\title{
ATRIBUCIÓN DE RESPONSABILIDAD ANTE LA VIOLENCIA SEXUAL: EFECTO DEL TIPO DE TÁCTICA, EL GÉNERO Y EL SEXISMO BENÉVOLO
}

\section{ATTRIBUTION OF RESPONSIBILITY FOR SEXUAL VIOLENCE: EFFECT OF TYPE OF TACTIC, GENDER AND BENEVOLENT SEXISM}

\section{MARTA GARRIDO-MACÍAS ${ }^{1}$, INMACULADA VALOR-SEGURA ${ }^{1} \mathrm{Y}$ FRANCISCA EXPÓSITO ${ }^{1}$}

\section{Cómo referenciar este artículo/How to reference this article:}

Garrido-Macías, M., Valor-Segura, I. y Expósito, F. (2017). Atribución de responsabilidad ante la violencia sexual: Efecto del tipo de táctica, el género y el sexismo benévolo [Attribution of responsibility for sexual violence: Effect of type of tactic, gender and benevolent sexism]. Acción Psicológica, 14(2), 69-84. https://doi.org/10.5944/ap.14.2.20757

\section{Resumen}

La violencia sexual es un problema actual sobre el que se necesita estudio empírico, pues afecta a más del $30 \%$ de las mujeres en sus relaciones con los hombres. El obje- tivo de esta investigación ha sido comprobar el modo en el que el tipo de táctica utilizada para conseguir sexo, el género y el sexismo benévolo influyen en la responsabilidad que se le atribuye al agresor. Utilizando una muestra de 305 participantes, se presenta un escenario sexual en el contexto de una relación de pareja, en el que se manipula el tipo de táctica utilizada para tener sexo (neutra, persuasión o coerción sexual). Los resultados indican que

\footnotetext{
Agradecimientos: Esta investigación fue financiada por el Ministerio de Economía y Competitividad de España, con el proyecto "Análisis psicosocial del acoso sexual: nuevas formas y nuevos contextos" (Ref. PSI2014-59200-R), así como por el Ministerio de Educación, Cultura y Deporte de España, con una beca de doctorado (FPU12/02905) para la primera autora de este artículo
}

Correspondencia: Marta Garrido-Macías, Centro de Investigación, Mente, Cerebro y Comportamiento, (CIMCYC). Universidad de Granada, Campus de Cartuja s/n, 18071. Granada, España. Email: martagarrido@ugr.es

ORCID: Marta Garrido-Macías (http://orcid.org/0000-0002-0522-7461), Inmaculada Valor-Segura (http://orcid.org/0000-0003-2303-3326) y Francisca Expósito (http://orcid.org/0000-0001-6157-4292).

${ }^{1}$ Universidad de Granada, España.

Recibido: 22 junio de 2017.

Aceptado: 09 de septiembre de 2017. 
las personas responsabilizan más al agresor cuando la táctica utilizada es persuasión o coerción sexual, que cuando se utilizan tácticas no violentas (neutra). Además, se comprueba que los hombres con alto sexismo benévolo culpan menos al agresor solamente cuando éste utiliza tácticas más sutiles de violencia sexual (persuasión sexual), mientras que este efecto no ocurre en las mujeres. Estos resultados permiten proporcionar un marco teórico sobre el que incidir para establecer los límites de los comportamientos que se consideran aceptables o no en una relación, contribuyendo a su detección y rechazo, así como para considerar su influencia en la toma de decisión del ámbito jurídico en los casos de violencia de género.

Palabras clave: Violencia sexual; Atribución de responsabilidad; Sexismo benévolo; Coerción sexual.

\section{Abstract}

Sexual violence is a current problem that requires empirical study, since it affects more than $30 \%$ of women in their relationships with men. The aim of the research has been to verify the way in which the type of tactics used to obtain sex, along with gender and benevolent sexism can have an impact on the responsibility attributed to the aggressor. Using a sample of 305 participants, a sexual scenario is presented in a relationship context where the type of tactic used to have sex (neutral, sexual coaxing, or sexual coercion) is manipulated. The results indicate that people attribute more responsibility to the aggressor when the tactic used is sexual coaxing or sexual coercion than when using non-violent (neutral) tactics. In addition, men with high benevolent sexism blame the aggressor less, but only when the subtlest tactics of sexual violence (sexual coaxing) are used, whereas this effect does not occur in women. These results provide a theoretical framework for establishing the limits of the behaviours that are considered acceptable (or not) in a relationship, contributing to their detection and having implications for legal decision-making in cases of gender violence.

Keywords: Sexual violence; Attribution of responsibility; Benevolent sexism; Sexual coercion.

\section{Introducción}

Pese a los cambios sociales acontecidos en España en las últimas décadas, seguimos viviendo en una sociedad patriarcal que subyuga a la mujer en muchos ámbitos, sociales, laborales, y también en la relación de pareja. Esta asimetría de poder que las mujeres tienen en su relación con los hombres, aumenta la vulnerabilidad a padecer cualquier situación de discriminación y violencia, pero, sobre todo, a aquellos tipos de violencia menos visibles, como es la violencia sexual (Kuyper, de Wit, Smolenski, Adam, Woertman y van Berlo, 2013).

El objetivo principal que subyace a la violencia sexual en la pareja es conseguir tener sexo con una persona que no está dispuesta, utilizando para ello diferentes tipos de tácticas que varían en función de su gravedad. Así, mientras las tácticas de agresión sexual que son mucho más graves incluyen el uso de fuerza física (DeGue y DiLillo, 2005; Katz y Tirone, 2009; McGregor, 2005), las tácticas de coerción sexual se consideran de menor gravedad, al utilizar la manipulación verbal y psicológica como forma de conseguir sexo (Black et al., 2011; DeGue y DiLillo, 2005). Finalmente, en el extremo más sutil, se encontrarían las tácticas de persuasión sexual, que incluyen tácticas benignas y seductivas para conseguir sexo (Camilleri, Quinsey y Tapscott, 2009).

Numerosos estudios han mostrado las consecuencias negativas para las víctimas que han sufrido violencia sexual, ya sean víctimas de agresión sexual, como de coerción o persuasión sexual. Entre estas consecuencias destacan: problemas de salud física (alteración del sueño, enfermedades de transmisión sexual, embarazos no deseados, disfunción sexual, etc.); problemas psicológicos (ansiedad, humillación, depresión, estrés, ideación suicida, síntomas de estrés postraumático, etc.); y problemas de comportamiento (abuso de sustancias, trastornos de alimentación, absentismo escolar o laboral, etc.) (Postma, Bicanic, van der Vaart y Laan, 2013; Ullman, Relyea, Peter-Hagene y Vasquez, 2013; Zinzow et al., 2012). Además, se ha comprobado que la violencia más sutil tiene un alto índice de prevalencia en las relaciones de pareja (Katz y Tirone, 2010; Salwen y O'Leary, 2013), siendo más utilizada que la más severa (RubioGaray, López-González, Saúl y Sánchez-Elvira-Pania- 
gua, 2012). Por ejemplo, Vannier y O’Sullivan (2010) mostraron que el $59 \%$ de las mujeres se habían implicado en sexo no deseado con su pareja, y Sorenson, Joshi y Sivitz (2014) constataron que el $64.5 \%$ conocían a una o más mujeres que habían sido víctimas de coerción sexual en su relación.

Sin embargo, pese a su alta prevalencia y las múltiples consecuencias que la violencia sexual tiene para las mujeres que la sufren, hay poca investigación sobre el tema, especialmente sobre las formas más sutiles (Katz y Tirone, 2010; Salwen y O'Leary, 2013). Esto puede ser debido al hecho de que la violencia sexual verbal es a menudo considerada aceptable, sobre todo en parejas que tienen una historia de sexo consensuado, y que les puede llevar a creerse en la obligación de continuar aceptando en relaciones sexuales futuras (Katz y Myhr, 2008; Lazar, 2010). Además, tanto la coerción sexual como la persuasión sexual han recibido escasa atención en el ámbito jurídico, pues este tipo de violencia no constituye una categoría de ofensa legalmente reconocida como si ocurre con la agresión sexual. De hecho, en el contexto legal, este tipo de víctimas son percibidas como persuadidas bajo presión psicológica, lo que implica que son parcialmente responsables y tienen control sobre la situación (McGregor, 2005).

Tomando en cuenta la percepción que la sociedad actual y el ámbito jurídico tienen acerca de la violencia sexual cuando coexiste con el uso de fuerza física, este estudio se interesa en determinar cómo el tipo de táctica, el género y el sexismo benévolo podrían estar influyendo en la responsabilidad que se le atribuye al agresor.

En primer lugar, se ha demostrado que el tipo de táctica utilizada influye en la responsabilidad atribuida a las partes. Así, las personas atribuyen más responsabilidad a la víctima cuando ésta ha sufrido violencia más leve que cuando ha sufrido violencia sexual con el uso de fuerza física (Katz, Moore y Tkachuk, 2007). Además, cuando el comportamiento del agresor incluye tácticas sexuales más violentas se percibe como más inaceptable y más negativo que cuando no incluye agresión (Capezza y Arriaga, 2008a, 2008b), responsabilizándolo más por su conducta.
En segundo lugar, los estudios centrados en las diferencias de género han comprobado que los hombres atribuyen menos responsabilidad al perpetrador que las mujeres (Basow y Minieri, 2011; Davies y Rogers, 2009). Por ejemplo, Byers y Glenn, (2012) comprobaron, utilizando una muestra de 140 participantes menores de 30 años que habían sufrido uno o más incidentes de coerción sexual desde los 14 años, que las mujeres responsabilizaban más al perpetrador que los hombres. Además, Vidal-Fernández y Megías (2014) han demostrado que los hombres culpan más a las víctimas de violencia física por parte de su pareja que las mujeres. Sin embargo, otros estudios no han encontrado diferencias significativas entre hombres y mujeres en la percepción de responsabilidad del agresor (Herrera, Pina, Herrera y Expósito, 2014; Tamborra, Dutton y Terry, 2014).

Además, algunas investigaciones han analizado la interacción del tipo de táctica y el género en la atribución de responsabilidad, encontrando que los hombres perciben a las víctimas de violencia sexual leve como más responsables que a las víctimas de violencia sexual grave, mientras que las mujeres no difieren en la responsabilidad atribuida en función del tipo de táctica (Katz et al., 2007; Rogers, Davies y Cottam, 2010).

Por último, la ideología sexista ejerce una influencia negativa en la compresión y percepción de la violencia de género (Durán, Campos-Romero y Martínez-Pecino, 2014; Herrera, Herrera y Expósito, 2014; Herrera, Herrera y Expósito, 2017) y en la reacción a la violencia sexual (Yamawaki, 2007). Se ha comprobado que las tácticas de violencia sexual más leves parecen estar más asociadas a actitudes permisivas o estereotípicas sobre el sexo y la mujer (DeGue y DiLillo, 2005). La ideología sexista se refiere a un conjunto de creencias sobre los roles de género, características y comportamientos que son considerados apropiados para hombres y mujeres, así como creencias sobre las relaciones entre ambos (Glick y Fiske, 1996). Esta ideología apoya las desigualdades de poder, generando un contexto propicio para la justificación de la dominación de los hombres sobre las mujeres. La teoría del sexismo ambivalente incluye tanto el sexismo hostil como el sexismo benévolo, siendo el sexismo benévolo el que comúnmente se ha asociado a la responsabilidad atribuida en casos de violencia de género 
(Durán, 2010; Valor-Segura, Expósito y Moya, 2011). Así, la literatura ha demostrado que adherirse a los roles de género tradicionales influye en la atribución de responsabilidad al agresor (Soto-Quevedo, 2012), de forma que las personas con alto sexismo benévolo culpan más a la víctima y exoneran más al perpetrador cuando la víctima se comporta de manera inconsistente con los roles de género tradicionales (Capezza y Arriaga, 2008a; Masser, Lee y Mckimmie, 2010; Pedersen y Siseñtrömwall, 2013; Soto-Quevedo, 2012; Valor-Segura et al., 2011; Vidal-Fernández y Megías, 2014; Yamawaki, 2007), tal y como se percibe cuando la víctima sufre violencia sexual dentro de su pareja o cuando ésta no se resiste.

Con todo lo expuesto, la presente investigación tiene como finalidad analizar cómo el tipo de táctica y el género influyen en la responsabilidad que se le atribuye al agresor, así como comprobar el papel del sexismo benévolo en dicha percepción. Para tal fin, se establecen las siguientes hipótesis:

H1: Se espera que se responsabilice más al agresor cuando se utilicen tácticas de violencia sexual más graves que cuando se utilicen tácticas más leves.

$\mathrm{H} 2$ : Se espera que las mujeres atribuyan más responsabilidad al agresor que los hombres.

H3: Se espera un efecto del sexismo benévolo sobre la responsabilidad atribuida al agresor en función del tipo de táctica y el género, de forma que un alto sexismo benévolo lleve a una menor atribución de responsabilidad del agresor cuando la táctica utilizada sea la persuasión y sólo en los participantes hombres.

\section{Método}

\section{Participantes}

La muestra estuvo compuesta por 305 estudiantes universitarios, 115 hombres (37.7\%) y 190 mujeres (62.3\%), con edades comprendidas entre los 18 y los 35 $(M=21.53, D T=3.23)$. De la muestra analizada, el $30.2 \%$ habían vivido una situación similar a la descrita y el $64.6 \%$ conocían a alguien que había vivido algo parecido a la situación descrita.

\section{Procedimiento y diseño}

Para esta investigación se ejecutó un diseño factorial completo 3 (tipo de táctica: neutra, persuasión y coerción) x 2 (género: masculino vs. femenino) con la responsabilidad atribuida al agresor como variable dependiente. Además, también se ejecutó una regresión por pasos, utilizando como variables independientes el sexismo benévolo, el tipo de táctica y el género; y como variable dependiente la responsabilidad atribuida al agresor.

La selección de los participantes se llevó a cabo mediante un muestreo incidental en las bibliotecas de las facultades de Psicología, Empresariales y Económicas, Farmacia y Relaciones Laborales de la Universidad de Granada. Una vez recogida la muestra, los sujetos accedieron a participar en el estudio de forma voluntaria rellenando un consentimiento informado en el que se les proporcionaba la información relativa al experimento y se les garantizaba el anonimato y la confidencialidad.

\section{Instrumentos de medida}

Se diseñó un instrumento formado por varias escalas en las que se les preguntaba a los participantes una serie de constructos relacionados con el objeto de investigación. Para introducir la manipulación experimental se presentaba la descripción de un escenario (ver apéndice) en el que se producía una relación sexual modificándose el tipo de táctica utilizada (neutra vs. coerción sexual vs. persuasión sexual), distribuyéndose los participantes a cada una de las tres condiciones de manera aleatoria. A continuación, se preguntaba por una serie de cuestiones relativas al episodio descrito.

- Percepción de violencia sexual: se utilizó la subescala de Tamborra et al. (2014), formada por 6 ítems que evalúan la percepción de violencia sexual de la situación descrita (e.g., "Ana se sintió presionada para tener sexo"), con un formato de respuesta tipo Líkert entre 1 
(totalmente en desacuerdo) y 5 (totalmente de acuerdo). Con los participantes en este estudio, la escala se mostró consistente internamente $(\alpha=.77)$.

- Responsabilidad del agresor: un ítem evalúa percepción de responsabilidad del agresor ("Antonio es más responsable de haber tenido sexo que Ana"), con un formato de respuesta tipo Líkert entre 1 (totalmente en desacuerdo) y 5 (totalmente de acuerdo).

- Escala de Sexismo Ambivalente (ASI) (Expósito, Moya y Glick, 1998). Para esta investigación se ha utilizado la subescala de sexismo benévolo, formada por 11 ítems (e.g., "las mujeres deben ser queridas y protegidas por los hombres") que presentan un formato de respuesta tipo Likert de 0 (totalmente en desacuerdo) a 5 (totalmente de acuerdo). Con los participantes en este estudio, la escala se mostró consistente internamente $(\alpha=.88)$.

- Características sociodemográficas. Se recogieron datos relativos al género y a la edad.

\section{Resultados}

\section{Manipulation check}

Con el objetivo de comprobar que se ha producido correctamente la manipulación experimental, se llevó a cabo un ANOVA utilizando como variable independiente el tipo de táctica (neutra vs. coerción vs. persuasión) y como variable dependiente la percepción de violencia sexual. Los resultados indican un efecto del tipo de táctica sobre la percepción de violencia sexual $F(2$, $302)=19.97, p<.001, \eta_{p}^{2}=.12$, encontrándose mediante la prueba de Tukey diferencias entre las tres tácticas. Así, se percibe una mayor violencia sexual en la condición de coerción $\left(M_{\text {coerción }}=3.77, D T_{\text {coerción }}=0.07\right)$, que en la de persuasión $\left(M_{\text {persuasión }}=3.54, D T_{\text {persua- }}\right.$ sión $=0.07)(p=.049)$ y que en la neutra $\left(M_{\text {neutra }}=3.08\right.$, $\left.D T_{\text {neutra }}=0.09\right)(p<.001)$, y una mayor violencia sexual en la condición de persuasión que en la neutra $(p<.001)$.

\section{Responsabilidad atribuida al agresor en función del tipo de táctica}

Para comprobar la Hipótesis 1 que afirmaba que las personas atribuirán más responsabilidad al agresor en función de la gravedad de la táctica utilizada, se llevó a cabo un ANOVA utilizando como variable independiente el tipo de táctica y como variable dependiente la responsabilidad atribuida al agresor. Los resultados indican un efecto del tipo de táctica sobre la responsabilidad atribuida, $F(2,302)=5.07, p=.007, \eta_{p}^{2}=.032$, encontrándose mediante la prueba de Tukey diferencias entre la condición neutra y las otras dos condiciones (coerción y persuasión). Así, como se observa en la Figura 1 se comprueba que se le atribuye más responsabilidad al agresor

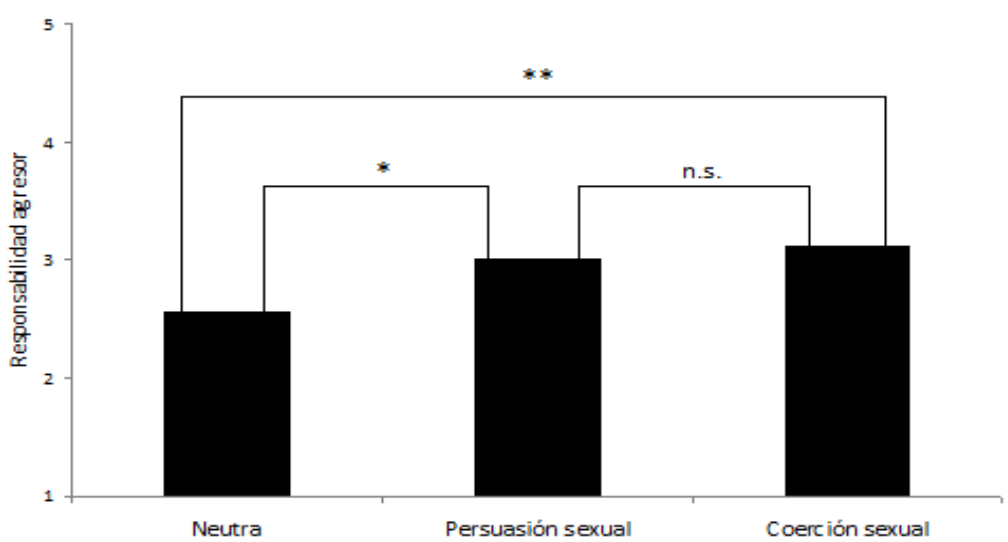

Figura 1. Responsabilidad atribuida al agresor en función del tipo de táctica ${ }^{* *} p<.01,{ }^{*} p<.05$. 
Tabla 1

Efecto del tipo de táctica, el género y el sexismo benévolo en la responsabilidad atribuida al agresor

\begin{tabular}{|c|c|c|c|c|}
\hline & & & $\mathrm{d}$ atrib & \\
\hline & & $\beta$ & $t$ & $p$ \\
\hline Paso 1 & Táctica & .13 & .78 & .435 \\
\hline & SB & -.41 & -1.5 & .149 \\
\hline & Género & -.04 & -.12 & .902 \\
\hline & $R^{2}$ & .03 & & \\
\hline Paso 2 & Táctica x SB & .21 & 1.14 & .255 \\
\hline & Táctica x Género & .08 & .36 & .721 \\
\hline & SB x Género & .75 & 2.21 & .028 \\
\hline & $R^{2}$ & .04 & & \\
\hline & $\Delta R^{2}$ & .02 & & \\
\hline Paso 3 & Táctica x SB x Género & -.52 & -2.30 & .022 \\
\hline & $R^{2}$ & .06 & & \\
\hline & $\Delta R^{2}$ & .03 & & \\
\hline
\end{tabular}

en la condición de coerción $\left(M_{\text {coerción }}=3.12, D T_{\text {coer- }}\right.$ ción $=0.11)$ que en la condición neutra $\left(M_{\text {neutra }}=2.56\right.$, $\left.D T_{\text {neutra }}=0.14\right)(p=.006)$ y en la condición de persuasión $\left(M_{\text {persuasión }}=3.01, D T_{\text {persuasión }}=0.11\right)$ que en la condición neutra $(p=.036)$.

\section{Diferencias de género en responsabili- dad del agresor}

Con el objetivo de verificar la Hipótesis 2 que afirmaba que las mujeres atribuyen más responsabilidad al agresor que los hombres, se llevó a cabo una $t$ de Student para muestras independientes, utilizando como variable independiente el género de los participantes y como variable dependiente la atribución de responsabilidad al agresor. Los resultados indican que no existen diferencias de género en la responsabilidad atribuida al agresor $t(303)=0.23, p=.822$, de forma que tanto hombres $(M=2.97, \quad D T=1.30) \quad$ como mujeres $\quad(M=2.93$, $D T=1.19)$ le atribuyen el mismo grado de responsabilidad.
Efecto del sexismo benévolo sobre la responsabilidad del agresor en función del tipo de táctica y el género

Con el objetivo de comprobar la Hipótesis 3, en la que se espera un efecto del sexismo benévolo, el tipo de táctica y el género sobre la responsabilidad atribuida al agresor, se llevó a cabo un análisis de regresión múltiple. Las variables independientes introducidas fueron el sexismo benévolo $(\mathrm{SB})$, el tipo de táctica $(0=$ neutra, $1=$ persuasión, $2=$ coerción $)$ y el género $(0=$ masculino, $1=$ femenino), y como variable dependiente se utilizó la responsabilidad atribuida al agresor. En el primer paso se contrastaron los efectos principales de las variables de interés, en el segundo paso las interacciones de segundo orden y en el tercer paso las interacciones de tercer orden. Los resultados se muestran en la Tabla 1.

De acuerdo con la Hipótesis 3, los resultados muestran una interacción significativa entre el sexismo benévolo, el tipo de táctica y el género (Figura 2). Tal y como se esperaba, el sexismo benévolo predice la responsabilidad atribuida al agresor sólo cuando la táctica utilizada es menos grave (persuasión) en el caso de los hombres $(b=-.35, E E=.18, p=.054)$, no obteniéndose efecto en la condición neutra $(b=-.03, E E=.56$, $p=.965)$ ni en la condición de coerción sexual $(b=.08$, 


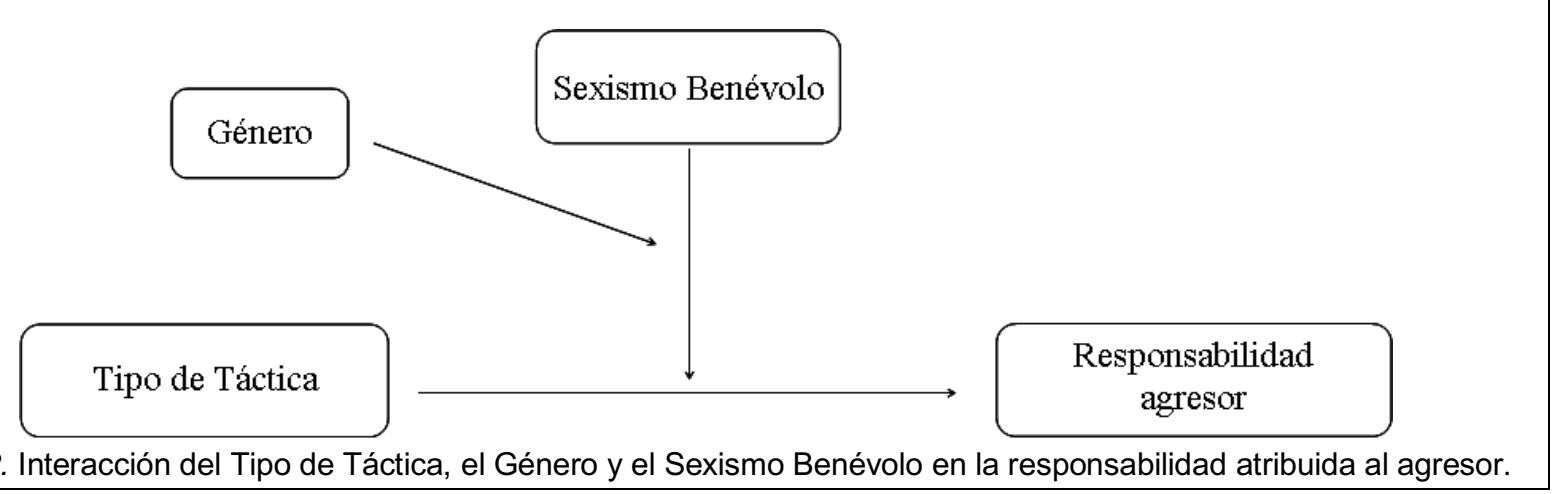

$E E=.19, p=.667)$. A su vez, no se encontró efecto del sexismo benévolo en las mujeres, ni en la condición neutra $(b=.42, E E=.27, p=.126)$; ni en la condición de persuasión $(b=-.08, E E=.14, p=.544)$; ni en la condición de coerción sexual $(b=-.22, E E=.13, p=.093)$.

\section{Discusión}

El objetivo fundamental de esta investigación consistió en comprobar cómo el tipo de táctica sexual utilizada por el agresor, el género y el sexismo benévolo de los observadores influyen en la responsabilidad que se atribuye al agresor.

En primer lugar, los resultados demuestran que cuando la relación sexual se lleva a cabo con el uso de tácticas que no implican violencia (neutras), las personas responsabilizan menos al agresor que cuando éste utiliza tácticas de persuasión sexual o de coerción sexual. Sin embargo, no existe una diferencia en la responsabilidad atribuida al agresor cuando se compara el uso de persuasión sexual y de coerción sexual, por lo que la Hipótesis 1 sólo puede ser aceptada parcialmente. Estos hallazgos permiten comprobar que, a pesar de que estudios previos demuestran una mayor responsabilidad atribuida al agresor cuando se han utilizado tácticas más graves como la fuerza física que cuando las tácticas han sido más sutiles (Capezza y Arriaga, 2008a; 2008b; Katz et al., 2007), las personas, en general, culpan al agresor en igual medida cuando éste utiliza cualquier tipo de tácticas que no implican el uso de la fuerza física, aunque difieran en gravedad (persuasión sexual vs. coerción sexual).

En segundo lugar, aunque algunos autores han encontrado que los hombres responsabilizan menos al agresor que las mujeres (Bascow y Minieri, 2010; Byers y Glenn, 2012; Davies y Rogers, 2009), el presente estudio lleva a rechazar la Hipótesis 2, pues no se encuentran diferencias de género en la responsabilidad atribuida al agresor, similar a resultados obtenidos en estudios previos (Herrera et al., 2014; Tamborra et al, 2014). Esta ausencia de diferencias de género puede ser debida a que tanto hombres como mujeres se encuentran insertos en una cultura cuyo sistema de creencias podría legitimar directa o indirectamente el empleo de violencia física en relaciones de pareja (Valor-Segura et al., 2011), por lo que las actitudes sexistas hacia las mujeres no sólo son mantenidas por los hombres, sino también por las mujeres (Soto-Quevedo, 2012).

Finalmente, en relación a la influencia que el sexismo benévolo ejerce en la responsabilidad atribuida al agresor, los resultados indican que puntuaciones altas en sexismo benévolo llevan a culpar en menor medida al agresor (Capezza y Arriaga, 2008a; Pedersen y Strömwall, 2013; Yamawaki, 2007) sólo cuando el tipo de táctica utilizada es la persuasión sexual y los participantes son hombres. Estos resultados permiten aceptar la Hipótesis 3 y confirman los hallazgos de estudios previos que demuestran que los hombres responsabilizaban más a las víctimas cuando se trata de violencia leve (versus violen- 
cia grave), mientras que las mujeres no varían la atribución de responsabilidad al agresor en función del tipo de violencia (Katz et al., 2007; Rogers et al., 2010).

Pese a que la presente investigación aporta datos que van en la línea de lo esperado y animan a seguir investigando en esta dirección, existen ciertas limitaciones que tratarán de solventarse en investigaciones futuras. En primer lugar, el hecho de que los escenarios de coerción sexual y de persuasión sexual incluyan violencia sexual sutil, ha podido influir en que los participantes atribuyan el mismo grado de responsabilidad al agresor, por lo que en investigaciones futuras sería deseable aportar más información de la situación, así como situaciones de agresión sexual para comprobar si los participantes reaccionan de manera diferencial.

La violencia sexual, a pesar de ser descrita como una de las experiencias más degradantes y humillantes que una persona puede experimentar (Bourke, 2007; Mackinnon, 2006), ha recibido una menor atención que la violencia física o la psicológica. Además, se suma el hecho de que existe una ambigüedad asociada a cómo clasificar, medir y etiquetar el completo rango de actos sexuales obligados. Las dificultades para comprender y medir las experiencias de violencia sexual son mayores cuando los actos ocurren en el contexto de las relaciones de pareja, resultando prácticamente imposible definir los límites de la integridad corporal dentro del contexto de las relaciones íntimas (Logan, Walker y Cole, 2015). En este sentido, comprender como la culpa o responsabilidad es asignada al agresor por observadores externos tiene importantes implicaciones en el campo de la psicología forense, pues puede ser un factor importante en las decisiones tomadas por la policía, jueces y fiscales que trabajan en casos de violencia de género (Weiss, 2009).

\section{Referencias}

Basow, S. A. y Minieri, A. (2011). "You owe me": Effects of date cost, who pays, participant gender, and rape myth beliefs on perceptions of rape. Journal of Interpersonal Violence, 26, 479-497. https://doi.org/10.1177/0886260510363421

Black, M. C., Basile, K. C., Breiding, M. J., Smith, S. G., Walters, M. L., Merrick, M. T., ... y Stevens, M. R. (2011). The National Intimate Partner and Sexual Violence Survey (NISVS): 2010 Summary Report. Atlanta, GA: National Center for Injury Prevention and Control, Centers for Disease Control and Prevention.

Bourke, J. (2007). Rape: Sex, Violence, History. Berkley, CA: Shoemaker and Hoard.

Byers, E. S. y Glenn, S. A. (2012). Gender Differences in Cognitive and Affective Responses to Sexual Coercion. Journal of Interpersonal Violence, 27, 827-845.

https://doi.org/10.1177/0886260511423250

Camilleri, J. A., Quinsey, V. L. y Tapscott, J. L. (2009). Assessing the Propensity for Sexual Coaxing and Coercion in Relationships: Factor Structure, Reliability, and Validity of the Tactics to Obtain Sex Scale. Archives of Sexual Behavior, 38, 959973. https://doi.org/ 10.1007/s10508-008-9377-2

Capezza, N. M. y Arriaga, X. B. (2008a). Factors Associated with Acceptance of Psychological Aggression against Women. Violence against Women, $14, \quad 612-633$. https://doi.org/10.1177/1077801208319004

Capezza, N. M. y Arriaga, X. B. (2008b). You Can Degrade but you can't Hit: Differences in Perceptions of Psychological versus Physical Aggression. Journal of Social and Personal Relationships, 25, 225-245. https://doi.org/10.1177/0265407507087957 
Davies, M. y Rogers, P. (2009). Perceptions of Blame and Credibility toward Victims of Childhood Sexual Abuse: Differences across Victim Age, VictimPerpetrator Relationship, and Respondent Gender in a Depicted Case. Journal of Child Sexual Abuse, 18, 78-92. https://doi.org/10.1080/10538710802584668

DeGue, S. y DiLillo, D. (2005). "You would if you Loved Me": Toward an Improved Conceptual and Etiological understanding of Nonphysical Male Sexual Coercion. Aggression and Violent Behavior, 10 , 513-532.

https://doi.org/10.1016/j.avb.2004.09.001

Durán, M. (2010). Sexismo benévolo y violencia sexual: percepción social de la violación en relaciones intimas [Benevolent Sexim and Sexual Violence: Social Perception of Rape in Intimate Relationships] (Tesis Doctoral). Universidad de Granada, Granada (España).

Durán, M., Campos-Romero, I. y Martínez-Pecino, R. (2014). Obstáculos en la comprensión de la violencia de género: influencia del sexismo y la formación en género. [Obstacles towards Gender Violence Comprehension: Influence of Sexism and Academic Training in Gender Issues]. Acción Psicológica, 11, 97-106. https://doi.org/10.5944/ap.11.2.14177

Expósito, F., Moya, M. C. y Glick, P. (1998). Sexismo ambivalente: medición y correlatos. [Ambivalent Sexism: Measurement and Correlatos]. Revista de Psicología Social, 13, 159-169. https://doi.org/10.1174/021347498760350641

Glick, P. y Fiske, S. T. (1996). The Ambivalent Sexism Inventory: Differentiating Hostile and Benevolent Sexism. Journal of Personality and Social Psychology, 70, 491-512. https://doi.org/10.1037/0022-3514.70.3.491

Herrera, A., Pina, A., Herrera, M. C. y Expósito, F. (2014). ¿Mito o realidad? Influencia de la ideología en la percepción social del acoso sexual [Myth or Reality? Influence of Ideology in the Sexual
Harassment Perception]. Anuario de Psicología Jurídica, 24, 1-7. https://doi.org/10.1016/j.apj.2014.07.002

Herrera, M. C., Herrera, A. y Expósito, F. (2014). Stop Harassment! Men's Reactions to Victims' Confrontation. European Journal of Psychology Applied to Legal Context, 6, 45-52. https://doi.org/10.1016/j.ejpal.2014.06.006

Herrera, M. C., Herrera, A. y Expósito, F. (2017). To confront versus not to confront: Women's Perception of Sexual Harassment. European Journal of Psychology Applied to Legal Context. Advance online publication. https://doi.org/10.1016/j.ejpal.2017.04.002

Katz, J., Moore, J. A. y Tkachuk, S. (2007). Verbal Sexual Coercion and Perceived Victim Responsibility: Mediating Effects of Perceived Control. Sex Roles, 57, 235-247. https://doi.org/10.1007/s11199-007-9253-X

Katz, J. y Myhr, L. (2008). Perceived Conflict Patterns and Relationship Quality Associated with Verbal Sexual Coercion by Male Dating Partners. Journal of Interpersonal Violence, 23, 798-814. https://doi.org/10.1177/0886260507313949

Katz, J. y Tirone, V. (2009). Women's Sexual Compliance with male Dating Partners: Associations with Investment in Ideal Womanhood and Romantic Well-Being. Sex Roles, 60, 347-356. https://doi.org/10.1007/s11199-008-9566-4

Katz, J. y Tirone, V. (2010). Going along with it: Sexually Coercive Partner Behavior predicts Dating Women's Compliance with Unwanted Sex. Violence against Women, 16, 730-742. https://doi.org/10.1177/1077801210374867

Kuyper, L., de Wit, J., Smolenski, D., Adam, P., Woertman, L. y van Berlo, W. (2013). Gender Differences in Patterns of Experienced Sexual Coercion and Associated Vulnerability Factors among Young People in the Netherlands. Journal of 
Interpersonal Violence, $\quad 20, \quad 1-22$. https://doi.org/10.1177/0886260513488689

Lazar, R. (2010). Negotiating sex: The Legal Construct of Consent in Cases of Wife Rape in Ontario, Canada. Canadian Journal of Women and the Law, 22, 329-363. https://doi.org/10.3138/cjwl.22.2.329

Logan, T. K., Walker, R. y Cole, J. (2015). Silenced Suffering: The Need for a better Understanding of Partner Sexual Violence. Trauma, Violence, \& Abuse, 16, 111-135. https://doi.org/10.1177/1524838013517560

MacKinnon, C.A. (2006). Are Women Human? And other International Dialogues. Cambridge, MA: The Belknap Press of Harvard University Press.

Masser, B., Lee, K. y McKimmie, B. M. (2010). Bad Woman, Bad Victim? Disentangling the Effects of Victim Stereotypicality, Gender Stereotypicality and Benevolent Sexism on acquaintance Rape Victim Blame. Sex Roles, 62, 494-504. https://doi.org/10.1007/s11199-009-9648-y

McGregor, J. (2005). Is it rape? On Acquaintance Rape and Taking Women's Consent Seriously. Burlington, VT: Ashgate.

Pedersen, S. H. y Strömwall, L. A. (2013). Victim Blame, Sexism and Just-World Beliefs: A CrossCultural Comparison. Psychiatry, Psychology and Law, 20, 932-941. https://doi.org/10.1080/13218719.2013.770715

Postma, R., Bicanic, I., van der Vaart, H. y Laan, E. (2013). Pelvic Floor Muscle Problems mediate Sexual Problems in Young Adult Rape Victims. The Journal of Sexual Medicine, 10, 1978-1987. https://doi.org/ 10.1111/jsm.12196

Rogers, P., Davies, M. y Cottam, L. (2010). Perpetrator Coercion, Victim Resistance and Respondent Gender: Their Impact on Blame Attributions in a Hypothetical Child Sexual Abuse Case. Journal of
Aggression, Conflict and Peace Research, 2, 25-35. https://doi.org/10.5042/jacpr.2010.0334

Rubio-Garay, F., López-González, M. A., Saúl, L. A. y Sánchez-Elvira-Paniagua, A. (2012). Direccionalidad y expresión de la violencia en las relaciones de noviazgo de los jóvenes [Directionality and Violence Expression in Dating Relationships of Young People]. Acción Psicológica, $\quad 9, \quad 61-70$. https://doi.org/10.5944/ap.9.1.204

Salwen, J. K. y O'Leary, K. D. (2013). Adjustment Problems and Maladaptive Relational Style: A Mediational Model of Sexual Coercion in Intimate Relationships. Journal of Interpersonal Violence, 28 , 1969-1988. https://doi.org/10.1177/0886260512471079

Sorenson, S. B., Joshi, M. y Sivitz, E. (2014). A Systematic Review of the Epidemiology of Nonfatal Strangulation, a Human Rights and Health Concern. American Journal of Public Health, 104, 54-61. https://doi.org/10.2105/AJPH.2014.302191

Soto-Quevedo, O. A. (2012). The Role of Ambivalent Sexism and Transgression of Gender Stereotype on Attribution of Blame to Female Victims of Intimate Partner Violence. Acta Colombiana de Psicología, $15,135-148$.

Tamborra, T. L., Dutton, L. B. y Terry, K. J. (2014). Verbally Coerced Sex: Does she Have to Say "no"? Interpersonal Review of Victimology, 20, 227-241. https://doi.org/10.1177/0269758014521740

Ullman, S. E., Relyea, M., Peter-Hagene, L. y Vasquez, A. L. (2013). Trauma Histories, Substance Use Coping, PTSD, and Problem Substance Use among Sexual Assault Victims. Addictive Behaviors, 38, 2219-2223.

https://doi.org/10.1016/j.addbeh.2013.01.027

Valor-Segura, I., Expósito, F. y Moya, M. (2011). Victim Blaming and Exoneration of the Perpetrator in Domestic Violence: The Role of Beliefs in a just 
World and Ambivalent Sexism. The Spanish Journal of Psychology, 14, 195-206. https://doi.org/10.5209/rev_SJOP.2011.v14.n1.17

Vannier, S. A. y O'Sullivan, L. F. (2010). Sex without Desire: Characteristics of Occasions of Sexual Compliance in young Adults' Committed Relationships. Journal of Sex Research, 47, 429439. https://doi.org/10.1080/00224490903132051

Vidal-Fernández, A. y Megías, J. L. (2014). Attributions of Blame to Battered Women when they are Perceived as Feminists or as "difficult to deal with". The Spanish Journal of Psychology, 17, 1-10. https://doi.org/10.1017/sjp.2014.26

Weiss, K. G. (2009). "Boys Will Be Boys" and other Gendered Accounts. An Exploration of Victims' Excuses and Justifications for Unwanted Sexual Contact and Coercion. Violence against Women, 15(7), 810-834 https://doi.org/10.1177/1077801209333611

Yamawaki, N. (2007). Rape Perception and the Function of Ambivalent Sexism and Gender-Role Traditionality. Journal of Interpersonal Violence, 22, 406-423. https://doi.org/10.1177/0886260506297210

Zinzow, H. M., Resnick, H. S., McCauley, J. L., Amstadter, A. B., Ruggiero, K. J. y Kilpatrick, D. G. (2012). Prevalence and Risk of Psychiatric Disorders as a Function of Variant Rape Histories: Results from a National Survey of Women. Social Psychiatry and Psychiatric Epidemiology, 47, 893902. https://doi.org/ 10.1007/s00127-011-0397-1.

\section{Apéndice}

\section{Escenario Neutro}

Ana y Antonio son dos jóvenes universitarios que van a la misma facultad. Llevan saliendo juntos 6 meses. Una noche quedan para ir a casa de un amigo común y pasan la noche riendo, hablando y coqueteando. Después, Ana invita a Antonio a su piso para seguir charlando un rato más. Cuando llegan, Ana lo hace pasar a su habitación y empieza a besarle. Se sientan en la cama y continúan besándose y acariciándose. Al poco tiempo, Antonio desliza su mano hacia el pantalón de Ana y consigue desabrocharlo.

-¿Qué haces?-dice Ana mientras se separa de Antonio.

- ¿Sabes que eres lo más bonito que me ha pasado hace mucho tiempo? Te deseo Ana..., y sé que tú también me deseas a mi-, responde Antonio con una dulce sonrisa en el rostro.

-A mi también me gustas mucho, pero tengo miedo de que me hagas daño...-murmura Ana.

-Shhhhh (le hace un gesto llevándose el dedo a los labios para hacerla callar) ... Lo sé Ana, pero entre dos personas que se atraen tanto es inevitable que pase algo especial. Sólo quiero estar contigo, demostrarte lo que siento...- responde Antonio mientras la sigue besando en las manos y en el cuello.

-Sí, es inevitable sentir lo que sentimos...-balbucea Ana mientras responde a los apasionados besos de Antonio.

Finalmente, Ana y Antonio acaban acostándose juntos.

\section{Escenario Persuasión}

Ana y Antonio son dos jóvenes universitarios que van a la misma facultad. Llevan saliendo juntos 6 meses. Una noche quedan para ir a casa de un amigo común y pasan la noche riendo, hablando y coqueteando. Después, Ana invita a Antonio a su piso para seguir charlando un rato más. Cuando llegan, Ana lo hace pasar a su habitación y empieza a besarle. Antonio responde a los besos y poco a poco desliza su mano hacia el pantalón de Ana, pero ella lo para y vuelve a colocar su mano en su cintura. Se sientan en la cama y continúan besándose y acaricián- 
dose. Al poco tiempo, Antonio vuelve a insistir bajando la mano y consigue desabrochar el pantalón de Ana.

- ¿Qué haces? ¡Para! - dice Ana mientras se separa de Antonio.

-No te asustes Ana. ¿Sabes que eres lo más bonito que me ha pasado hace mucho tiempo? Te deseo Ana..., y sé que tú también me deseas a mi-responde Antonio con una dulce sonrisa en el rostro.

-Sólo quería pasar un rato contigo, nada más- murmura Ana.

-Shhhhh le hace un gesto llevándose el dedo a los labios para hacerla callar) ... Lo sé Ana, pero entre dos personas que se atraen tanto es inevitable que pase algo especial. Sólo quiero estar contigo, demostrarte lo que siento...- insiste Antonio mientras la sigue besando en las manos y en el cuello.

-Mmm... es que no me apetece, de verdad! ...balbucea Ana mientras responde, con desgana, a los apasionados besos de Antonio.

Tras los continuos argumentos de Antonio y sus insistentes caricias, Ana finalmente cede y se acuestan juntos.

\section{Escenario Coerción}

Ana y Antonio son dos jóvenes universitarios que van a la misma facultad. Llevan saliendo juntos 6 meses. Una noche quedan para ir a casa de un amigo común y pasan la noche riendo, hablando y coqueteando. Después, Ana invita a Antonio a su piso para seguir charlando un rato más. Cuando llegan, Ana lo hace pasar a su habitación y empieza a besarle. Antonio responde a los besos y poco a poco desliza su mano hacia el pantalón de Ana, pero ella lo para y vuelve a colocar su mano en su cintura. Se sientan en la cama y continúan besándose y acariciándose. Al poco tiempo, Antonio vuelve a insistir bajando la mano y consigue desabrochar el pantalón de Ana.

-¿Qué haces? ¡Para! - dice Ana mientras se separa de Antonio.

- iVenga ya Ana! No me gustaría irme de aquí así. Además, está claro que quieres algo más conmigo, igual que yo-responde Antonio con una sonrisa en el rostro.

-Sólo quería pasar un rato contigo, nada más- murmura Ana.

- Si solo quisieras eso, no me habrías traído a tu casa, ni estaríamos en tu habitación. Si de verdad sintieras por mí lo que yo siento por ti, no dudarías ni un segundo- in- siste Antonio mientras la sigue besando en las manos y en el cuello.

-Mmm... es que no me apetece, de verdad! ...balbucea Ana mientras responde, con desgana, a los apasionados besos de Antonio.

Tras los continuos argumentos de Antonio y sus insistentes caricias, Ana finalmente cede y se acuestan juntos. 


\title{
ATTRIBUTION OF RESPONSIBILITY FOR SEXUAL VIOLENCE: EFFECT OF TYPE OF TACTIC, GENDER AND BENEVOLENT SEXISM
}

\author{
MARTA GARRIDO-MACÍAS ${ }^{1}$, INMACULADA VALOR-SEGURA ${ }^{1}$ Y \\ FRANCISCA EXPÓSITO ${ }^{1}$
}

\section{EXTENDED SUMMARY}

\section{Introduction}

Sexual violence is one of the most humiliating and devastating forms of violence against women. Within the couple relationship, the main objective is to obtain sex with a person who is unwilling by using different types of tactics that can vary according to their degree of severity. Firstly, sexual aggression appears to be the most severe, including the use of direct and invasive physical force to obtain sex (DeGue \& DiLillo, 2005; Katz \& Tirone, 2009; McGregor, 2005). Secondly, sexual coercion is less severe than sexual aggression, because it includes the use of verbal and psychological manipulation as a means of obtaining sex (Black et al., 2011; DeGue \& DiLillo, 2005).

Finally, sexual coaxing tactics are located at the other extreme to sexual aggression, including the use of more subtle tactics that are more charming and benign $(\mathrm{Ca}$ milleri, Quinsey, \& Tapscott, 2009).Sexual violence has immeasurable consequences for the affected women, such as physical (sleep alterations, sexual dysfunction, etc.), psychological (anxiety, depression, etc.), and behavioural (substance abuse, eating disorders, etc.) problems (Postma, Bicanic, van der Vaart, \& Laan, 2013; Ullman, Relyea, Peter-Hagene, \& Vasquez, 2013; Zinzow et al., 2012). Further, it has been demonstrated that less severe violence is highly prevalent in couple relationships (Katz \& Tirone, 2010; Salwen \& O'Leary,
2013) being more commonly used than severe violence (Rubio-Garay, López-González, Saúl, \& Sánchez-ElviraPaniagua, 2012). For instance, Vannier and O'Sullivan (2010) showed that $59 \%$ of women have been involved in undesired sex with their partners, and Sorenson, Joshi and Sivitz (2014) noted that $64.5 \%$ know one or more women that have been victims of sexual coercion.

Although sexual violence has high prevalence and multiple consequences for the affected women, relatively few studies have investigated the subtlest forms of violence (Katz \& Tirone, 2010; Salwen \& O'Leary, 2013). This could be due to the fact that sexual violence can occasionally be normalised, particularly in couple relationships that have a history of consensual sex and in which there is the belief that they must continue to accept sex in future encounters (Katz \& Myhr, 2008; Lazar, 2010). Moreover, both sexual coercion and sexual coaxing have received little attention in the legal context because, unlike sexual aggression, they do not fall under any legally recognized category of offense. In fact, in the legal context, these types of victims are perceived as being subject to persuasion under psychological pressure, implying that they are partially responsible and have control over the situation (McGregor, 2005).

Given these current perceptions regarding sexual violence when it coexists with the use of physical force both in modern society in general and the legal context in particular-this study is concerned with determining how the type of tactics, gender, and benevolent sexism could 
have an impact on the responsibility attributed to the aggressor.

Firstly, with respect to the type of tactic, research has shown that people attribute more responsibility to the victim when they have suffered less severe violence than sexual violence with the use of physical force (Katz, Moore, \& Tkachuk, 2007). Moreover, the behaviour of the aggressor is more likely to be perceived as unacceptable and negative when he uses more violent sexual tactics than when he does not use aggression (Capezza \& Arriaga, 2008a; Capezza \& Arriaga, 2008b).

Secondly, some studies on gender differences have found that men attribute less responsibility to the aggressor than women (Basow \& Minieri, 2011; Byers \& Glenn, 2012; Davies \& Rogers, 2009), whilst others have not found significant differences (Herrera, Pina, Herrera, \& Expósito, 2014; Tamborra, Dutton, \& Terry, 2014). Some researchers have analysed the interaction between the type of tactic and gender on the attribution of responsibility. They have shown that men attribute more responsibility to victims of subtle sexual violence than victims of severe sexual violence, whereas women do not differ in the responsibility attributed depending on the type of tactic (Katz et al., 2007; Rogers, Davies, \& Cottam, 2010).

Finally, benevolent sexism has a negative influence on both the understanding and perception of gender violence (Durán, Campos-Romero, \& Martínez-Pecino, 2014; Herrera, Herrera, \& Expósito, 2014; Herrera, Herrera, \& Expósito, in press) and the reaction to more subtle sexual violence (Yamawaki, 2007). The literature has shown that assuming traditional gender roles has an influence on the attribution of responsibility to the aggressor (Soto-Quevedo, 2012). Thus, people with higher benevolent sexism place more blame on the victim and less on the aggressor when the victim's behaviour is incompatible with traditional gender roles (Capezza \& Arriaga, 2008a; Masser, Lee, \& Mckimmie, 2010; Pedersen \& Strömwall, 2013; Soto-Quevedo, 2012; Valor-Segura et al., 2011; Vidal-Fernández \& Megías, 2014).

In summary, this study focuses on analysing the effect of the type of sexual tactic and gender on the re- sponsibility attributed to the aggressor, as well as demonstrating the influence of benevolent sexism on this perception. Thus, we expected to find higher attribution of responsibility to the aggressor when the sexual tactics are more severe compared with the case in which more subtle tactics are employed (Hypothesis 1). Further, it is expected that women attribute more responsibility to the aggressor than men (Hypothesis 2). Finally, it is anticipated that benevolent sexism will have an impact on attributed responsibility depending on the type of tactic and gender; in particular, higher benevolent sexism will lead to a lower attribution of responsibility to the aggressor when the type of sexual tactic used is coaxing, but only in men (Hypothesis 3 ).

\section{Method}

\section{Participants}

The sample consisted of 305 students from the university of Granada, and was composed of 115 males $(37.7 \%)$ and 190 females $(62.3 \%)$, with an age range between 18 and 35 years $(M=21.53, S D=3.23)$.

\section{Procedure and design}

The study adopted a factorial design 3 (type of tactic: neutral, coaxing or coercion) x 2 (gender: masculine vs. femenine) with the responsibility attributed to the aggressor as the dependent variable. Further, a stepwise regression was conducted too, using benevolent sexism, type of tactic and gender as the independent variables; and the responsibility attributed to the aggressor as the dependent variable. An incidental sampling method was used to select the participants from some of the libraries in the University of Granada. All the participants were volunteers, and their information was kept confidential and anonymous, thereby complying with the university research ethics committee. 


\section{Instruments}

An instrument was designed to include the target measures. To introduce the experimental manipulation, the participants were presented with the description of a scenario in which a sexual relation occurred, manipulating the type of tactic used (neutral, coaxing, or coercion). The participants were randomly allocated to one of the three conditions, after which they were required to answer a series of questions related to the situation described (perception of sexual violence and responsibility attributed to the aggressor) as well as the subscale of benevolent sexism.

\section{Results}

\section{Effect of the type of tactic on the at- tribution of responsibility}

To test Hypothesis 1 an ANOVA was conducted on the data. This analysis revealed an effect of tactic on the attribution of responsibility, $F(2,302)=5.07, p=.007$, $\eta^{2}{ }_{p}=.032$, that is, following Tukey's test, differences were found between the neutral and coercion conditions $(p=.006)$, and also between neutral and coaxing $(p=.036)$. Thus, more responsibility is attributed to the aggressor when the type of tactic used is coaxing or coercion compared with the case in which the aggressor does not use sexual violence (neutral condition).

\section{Gender differences in responsibility of the aggressor}

In order to test Hypothesis 2 a Student's t-test was conducted using gender as the independent variable and attribution of responsibility to the aggressor as the dependent variable. This analysis revealed no gender differences in the responsibility attributed to the aggressor $t(303)=0.23, p=.822$.

\section{Effect of benevolent sexism on respon- sibility of the aggressor as a function of type of tactic and gender}

A moderated multiple regression analysis was employed to test Hypothesis 3. The independent variables were Benevolent Sexism (BS), type of tactic $(0=$ neutral vs. $1=$ coaxing vs. $2=$ coercion $)$, and gender $(0=$ men vs. $1=$ women). The dependent variable was the responsibility attributed to the aggressor. The results showed a significant three-way interaction between benevolent sexism, type of tactic, and gender. As expected, a simple effect test revealed that benevolent sexism predicted responsibility attributed to the aggressor only when the tactic used was sexual coaxing and only in the case of men, $(b=-.35, S E=.18, p=.054)$. Benevolent sexism was not a significant predictor of responsibility in the case of men when the tactic used was sexual coercion $(b=.08, S E=.19, p=.667)$ or neutral $(b=-.03$, $S E=.56, p=.965)$, or in the case of women when the tactic used was neutral $(b=.42, S E=.27, p=.126)$; sexual coaxing $(b=-.08, S E=.14, p=.544)$; or sexual coercion $(b=-.22, S E=.13, p=.093)$.

\section{Discussion}

This study set out to explore how, in a scenario of sexual violence, the type of tactic employed, gender, and benevolent sexism can all have an impact on the level of responsibility attributed to the aggressor.

First, the present findings demonstrate that people tend to attribute less responsibility to the aggressor when the tactics used to perform sexual violence in the couple relationship do not include violence (neutral) in comparison with the case in sexual coaxing or sexual coercion are used. However, there are no differences in the responsibility attributed to the aggressor when sexual coaxing and sexual coercion are compared, and thus Hypothesis 1 can only be partially accepted. These findings suggest that, although previous studies have demonstrated that more responsibility is attributed to the aggressor when more severe tactics are used in comparison with the use of more subtle tactics (Capezza \& Arriaga, 2008a; 2008b; Katz et al., 2007), people generally tend 
to blame the aggressor equally when he uses any type of tactics that do not involve the use of physical force, even though they differ in severity (sexual coaxing vs. sexual coercion).

Second, although some studies have found gender differences in the responsibility attributed to the aggressor (Bascow \& Minieri, 2010; Byers \& Glenn, 2012; Davies \& Rogers, 2009), results of the present study lead us to reject Hypothesis 2, since no gender differences have been found, which is in agreement with the results of previous studies (Herrera et al., 2014; Tamborra et al, 2014). This finding could be due to the fact both men and women are embedded in a culture whose belief system could directly or indirectly legitimize the use of physical violence in relationships (Valor-Segura et al., 2011). Thus, sexist attitudes toward women are not only maintained by men, but also by women (Soto-Quevedo, 2012).

Finally, regarding the influence of benevolent sexism on the responsibility attributed to the aggressor, the results show that people with higher benevolent sexism place less blame on the aggressor (Capezza \& Arriaga, 2008a; Pedersen \& Strömwall, 2013; Yamawaki, 2007) but only when the type of tactic used is sexual coaxing and only in the case of men. These findings support Hypothesis 3 and they agree with previous research demonstrating that men blame the victim more when subtle violence occurs (vs. severe violence), whereas women do not vary in their attribution of responsibility to the aggressor according to the type of violence (Katz et al., 2007; Rogers et al., 2010).

Sexual violence, in spite of being described as one of the most humiliating and devastating experiences that a person can suffer (Bourke, 2007; Mackinnon, 2006), has received relatively less attention than physical or psychological violence. Moreover, it is difficult to understand and to measure the experiences of sexual violence, particularly when these acts occur within the context of a romantic relationship (Logan, Walker \& Cole, 2015). In this regard, understanding how the blame or responsibility is assigned to the aggressor by external observers has important implications in the context of forensic psychology, since this could be an important factor in the decisions made by the police, judges, and fiscal authorities that work together on cases of gender violence (Weiss, 2009). 


\title{
INDICADORES DE CALIDAD DE LAS \\ PUBLICACIONES CIENTÍFICAS EN PSICOLOGÍA JURÍDICA
}

\author{
QUALITY INDICATORS OF SCIENTIFIC \\ PUBLICATIONS IN LEGAL PSYCHOLOGY
}

\author{
JULIA OSCA-LLUCH ${ }^{1}$, MACARENA TORTOSA ${ }^{2}$, \\ FRANCISCO GONZÁLEZ-SALA ${ }^{3}$ Y FRANCISCO TORTOSA ${ }^{3}$
}

Cómo referenciar este artículo/How to reference this article:

Osca-Lluch, J., Tortosa, M., González-Sala, F. y Tortosa, F. (2017). Indicadores de calidad de las publicaciones científicas en psicología jurídica [Quality Indicators of Scientific Publications in Legal Psychology]. Acción Psicológica, 14(2), 85-98. https://doi.org/10.5944/ap.14.2.20759

\section{Resumen}

Las publicaciones científicas son una de las dimensiones básicas de la evaluación de la calidad de la investigación del personal docente e investigador en España. El criterio más utilizado para la evaluación es la publicación de trabajos en revistas incluidas en las bases de datos de la Web of Science (WoS) o Scopus. El objetivo de este trabajo es conocer que revistas de psicología, que publican trabajos de psicología jurídica, son las que están actualmente incluidas en las bases de datos Web of Science
(WoS) y Scopus, ofrecer un análisis y comparación de los indicadores de calidad de las revistas en estas bases de datos, con el propósito de que los investigadores que trabajan en esta disciplina conozcan la situación de las publicaciones de su campo de conocimiento correspondiente y facilitarles la mejor elección para publicar.

Palabras clave: Revistas científicas; Psicología jurídica; Factor de impacto; Web of Science; JCR; Scopus; SJR.

Correspondencia: Julia Osca Lluch, Instituto de Gestión de la Innovación y del Conocimiento, INGENIO (CSIC-UPV), Universitat Politècnica de València, España. Email: juosllu@ingenio.upv.es

ORCID: Julia Osca Lluch (http://orcid.org/0000-0002-0449-5878), Macarena Tortosa (http://orcid.org/0000-0002-79004877), Francisco González-Sala (http://orcid.org/0000-0003-4124-7459) y Francisco Tortosa (http://orcid.org/0000-0003$\underline{3718-8441)}$.

${ }^{1}$ INGENIO (CSIC-UPV), Universitat Politècnica de València, España.

${ }^{2}$ Universidad Internacional de Valencia-VIU, España.

${ }^{3}$ Universitat de València, España.

Recibido: 05 mayo de 2017.

Aceptado: 18 de julio de 2017. 


\begin{abstract}
The scientific publications are one of the basic dimensions of the evaluation of the quality of research of teaching and research personnel in Spain. The most used criterion for the evaluation is the publication of papers in journals included in the Web of Science (WoS) or Scopus databases. The objective of this work is to know that psychology journals, which publish works of legal psychology, are currently included in the Web of Science (WoS) and Scopus databases, offer an analysis and comparison of the quality indicators of the journals in these databases, in order that researchers working in this discipline know the status of publications in their field of knowledge and provide them with the best choice to publish.
\end{abstract}

Keywords: Scientific journals; Legal psychology; Impact factor; Web of Science; JCR; Scopus; SJR.

\section{Introducción}

Las publicaciones científicas son el principal vehículo de difusión de la ciencia. Su estudio y evaluación está alcanzando un importante desarrollo en los últimos años en las diferentes áreas o disciplinas de la ciencia, por el papel tan relevante que desempeñan en el marco de la investigación científica, como herramientas que resultan fundamentales para la divulgación científica y que, además, posibilitan el avance del conocimiento y alcanzar la excelencia científica. Toda comunidad requiere de un medio de expresión a través del cual registra, transmite e intercambia experiencias entre sus propios miembros y con los de otros grupos. La cultura científica no puede existir al margen de las publicaciones científicas, pero no todas tienen el mismo prestigio y grado de influencia en la comunidad científica. Su reconocimiento depende en gran medida de su calidad y su visibilidad (Osca-Lluch, 2012).

Las revistas científicas definen el "research front" de cualquier disciplina académica. Existe una interacción característica entre nivel de desarrollo institucional e implantación social y número y diversidad de revistas.
Cuando se ofrecen revisiones del estado actual de cualquier ciencia o ámbito de la misma, en general, el protagonismo de los artículos de revista es absoluto. Los revisores recurren a éstas para definir las áreas más importantes y novedosas de investigación y aplicación, y los conceptos, paradigmas, métodos y tecnologías dominantes y definitorias de eso que muchos llaman el "state of art". Incluso es habitual encontrar, en la literatura historiográfica, referencias al singular papel de las revistas en el proceso institucionalizador. Su existencia o inexistencia ayuda a explicar grados de institucionalización y su diversificación es fiel reflejo de la creciente superespecialización que acompaña al desarrollo disciplinar (Quiñones, Peñaranda, López y Tortosa, 2004; Tortosa y Civera, 2001; Tortosa, Santolaya y Civera, 2015). Se puede seguir la actividad de los investigadores o de un departamento o área científica analizando los artículos que publican en revistas científicas (Buela-Casal, Bermúdez, Sierra, Guillén-Riquelme y Quevedo-Blasco, 2015). El análisis de los trabajos da indicaciones sobre el dinamismo de un campo o disciplina científica, sobre su producción científica y sobre los hábitos de publicación y difusión de los investigadores.

Sin embargo, no todas las revistas poseen la misma relevancia como medio para la difusión de la producción investigadora. La escasa visibilidad de las publicaciones científico-técnicas, particularmente de las que se editan en español, afecta a los investigadores cuando son evaluados en el área de sus publicaciones. Así, en este contexto, se observa una tendencia, entre las instituciones y agencias que evalúan la producción de los científicos, a minusvalorar, cuando no a ignorar, las publicaciones de sus trabajos en revistas que no están incluidas en bases de datos internacionales. Por esta razón, la mayor o menor disposición de los autores a publicar en una revista científica, viene influenciada por el grado de visibilidad internacional de la misma, el cual a su vez depende de la presencia en bases de datos bibliográficas internacionales de prestigio.

Uno de los medios para conocer el estado de la actividad investigadora y docente de cualquier institución, consiste en la evaluación de la calidad de las revistas donde publican sus integrantes, aumentando su prestigio en función del factor de impacto que poseen éstas, inde- 
pendientemente del nivel de excelencia del contenido del trabajo publicado (Navarrete-Cortés, Quevedo-Blasco, Chaichio-Moreno, Ríos y Buela-Casal, 2009).

La importancia que la psicología jurídica ha cobrado en los últimos años es un hecho evidente si consideramos su evolución no solo a nivel científico sino también en el terreno aplicado, siendo hoy en día una parte inseparable en el derecho y en la criminología. La Psicología Jurídica ha sido entendida como la aplicación de la ciencia psicológica al Derecho, si bien, tal y como apunta Arce (2005) la Psicología Jurídica no solo cuenta con una parte aplicada, sino que también es capaz de elaborar teorías, metodologías que condicionan las actuaciones propias del Derecho y comprende más allá de éste, al abordar aspectos relevantes y relacionados con el comportamiento humano previo a la comisión de un acto delictivo. Psicología y Derecho aúnan posiciones teóricas, metodológicas y aplicadas para entender los factores propios de las personas, a nivel social, psicológico, conductual, relacional, de salud, entre otros, que afectan o pueden afectar al comportamiento legal de todos los actores que intervienen en el proceso de forma directa como jurados, jueces, peritos, testigos, víctimas, agresores, investigadores, cuerpos de seguridad, o de forma indirecta como grupos de riesgo o la población en general en los estudios relacionados con prevalencia de conductas delictivas, la prevención del riesgo o la influencia de la sociedad, del barrio o de la familia en determinados comportamientos que pueden conducir a un proceso legal (González-Sala, Osca-Lluch, Tortosa y Peñaranda, 2017). Por este motivo, la psicología jurídica tiene un papel fundamental en la toma de decisiones judiciales (Amado, Arce y Fariña, 2015; Amado, Arce, Fariña y Vilariño, 2016; Arce, Fariña y Fraga, 2000) y en la resolución de temas tan lamentables y frecuentes como la violencia doméstica y malos tratos tanto en adultos (Arce, Fariña y Vilariño, 2015; Cala, Trigo y Saavedra, 2016; Contreras y Cano, 2016) como en adolescentes (Álvarez-García, Barreiro-Collazo, Núñez y Dobarro, 2016).

El objetivo de este trabajo es conocer que revistas de psicología, que publican trabajos de psicología jurídica, están actualmente incluidas en las bases de datos Web of Science (WoS) y Scopus y ofrecer un análisis de los in- dicadores de calidad de las revistas en estas bases de datos, con el propósito de que los investigadores que trabajan en esta disciplina conozcan la situación de las publicaciones de su campo de conocimiento correspondiente y facilitarles la mejor elección para publicar.

\section{Material y método}

Este estudio utiliza como fuente de información las bases de datos Journal Citation Reports (Social Sciences Edition y Science Edition), en adelante, JCR, de la empresa Thompson Reuters y el Scimago Journal \& Country Rank (SJR), base de datos que ofrece diversa información e indicadores bibliométricos de las revistas incluidas en la base de datos Scopus, del grupo Elsevier.

Tanto el JCR (SCI y SSCI), como el SJR, clasifican las revistas según una lista de materias que ellos mismos elaboran. En el caso de la psicología, algunas revistas pueden encontrarse indizadas en una, dos o incluso en las tres bases de datos. También pueden estar incluidas en diferentes categorías temáticas, incluso dentro de la misma base de datos, lo que da lugar a que una revista ocupe diferentes posiciones y cuartiles, según la categoría temática en la que este.

Cuando una misma revista se encuentra clasificada en dos o más categorías temáticas diferentes en una base de datos, se considera que esas categorías tienen alguna relación y se puede, a partir de las mismas, detectar relaciones entre las diferentes disciplinas. Estas relaciones existentes entre las diferentes categorías temáticas deberían de tenerse en cuenta, cuando se quieren realizar estudios sobre la producción científica de un grupo de investigación, área o disciplina científica, sobre todo si esta es multidisciplinar, ya que puede haber trabajos de nuestro interés publicados en revistas en categorías temáticas relacionadas con nuestro objeto de estudio.

En este trabajo, a partir de las revistas incluidas en la edición del 2015 de los JCR y SJR, se ha realizado un estudio bibliométrico sobre las revistas de psicología jurídica, que están recogidas en estas bases de datos, con el propósito de conocer que revistas están mejor posiciona- 
Tabla 1

País de edición y solapamiento entre las bases de datos

\begin{tabular}{|c|c|c|c|}
\hline Países & $\begin{array}{c}N^{\circ} \text { revistas solapadas } \\
\text { (JCR y SJR) }\end{array}$ & $N^{0}$ revistas solo SJR & Total revistas por país \\
\hline Australia & 1 & & 1 \\
\hline Croacia & 1 & & 1 \\
\hline Eslovenia & 1 & & 1 \\
\hline España & 1 & 1 & 2 \\
\hline Estados Unidos & 10 & 1 & 11 \\
\hline Holanda & 1 & & 1 \\
\hline Reino Unido & 11 & 1 & 12 \\
\hline Suiza & 1 & & 1 \\
\hline Total & 27 & 3 & 30 \\
\hline
\end{tabular}

das, dentro de esta disciplina, su posición, así como que países lideran la edición de revistas de psicología jurídica.

Se realizó una búsqueda en las bases de datos, seleccionando en cada una de ellas aquellas revistas que estuvieran clasificadas en alguna de las categorías de psicología y que, además también estuvieran clasificadas en alguna de las siguientes categorías temáticas: "Law", "Criminology \& Penology" o incluyera en el título de la publicación o en la institución editora los términos "Law", "Criminology", "Criminal", "Penology", "Forensic", "Legal". La información sobre las revistas seleccionadas procedente de las bases de datos, se descargaron en una base de datos, diseñada "ad hoc" para este estudio.

\section{Resultados}

\section{Cobertura y solapamiento de revistas entre JCR (SCI y SSCI) y SJR}

La consulta a las diferentes bases de datos consultadas, en su edición publicada en el año 2016 dio como resultado un total de 30 revistas. Su distribución por base de datos arrojó un número de revistas muy similar, en JCR (SCI y SSCI) se recuperaron 27 revistas y en SJR, 30.
De todas las revistas seleccionadas, hay que destacar que 27 de ellas, circulan en las bases de datos JCR y SJR, y que solamente hay tres revistas que únicamente están incluidas en el SJR, que son: American Journal of Forensic Psychology, Anuario de Psicología Jurídica y Journal of Forensic Practice.

Un aspecto que hay que mencionar es que, de las 27 revistas incluidas en el JCR, 11 de ellas no están clasificadas en ninguna de las categorías temáticas de psicología, pero que hemos seleccionado para nuestro estudio porque sí que están incluidas en la base de datos SJR.

\section{Cobertura de las bases de datos según país de edición de las revistas}

Cuando se analiza el país de procedencia de las revistas de psicología jurídica incluidas en las bases de datos JCR y SJR, se observa que están editadas por 8 países. Tal como se muestra en la Tabla 1, Reino Unido y Estados Unidos son los países que editan un mayor número de publicaciones, con 12 y 11 revistas respectivamente. El resto de países, solamente tiene una revista de psicología jurídica en el JCR y, en el caso de España, también una revista en el SJR. 


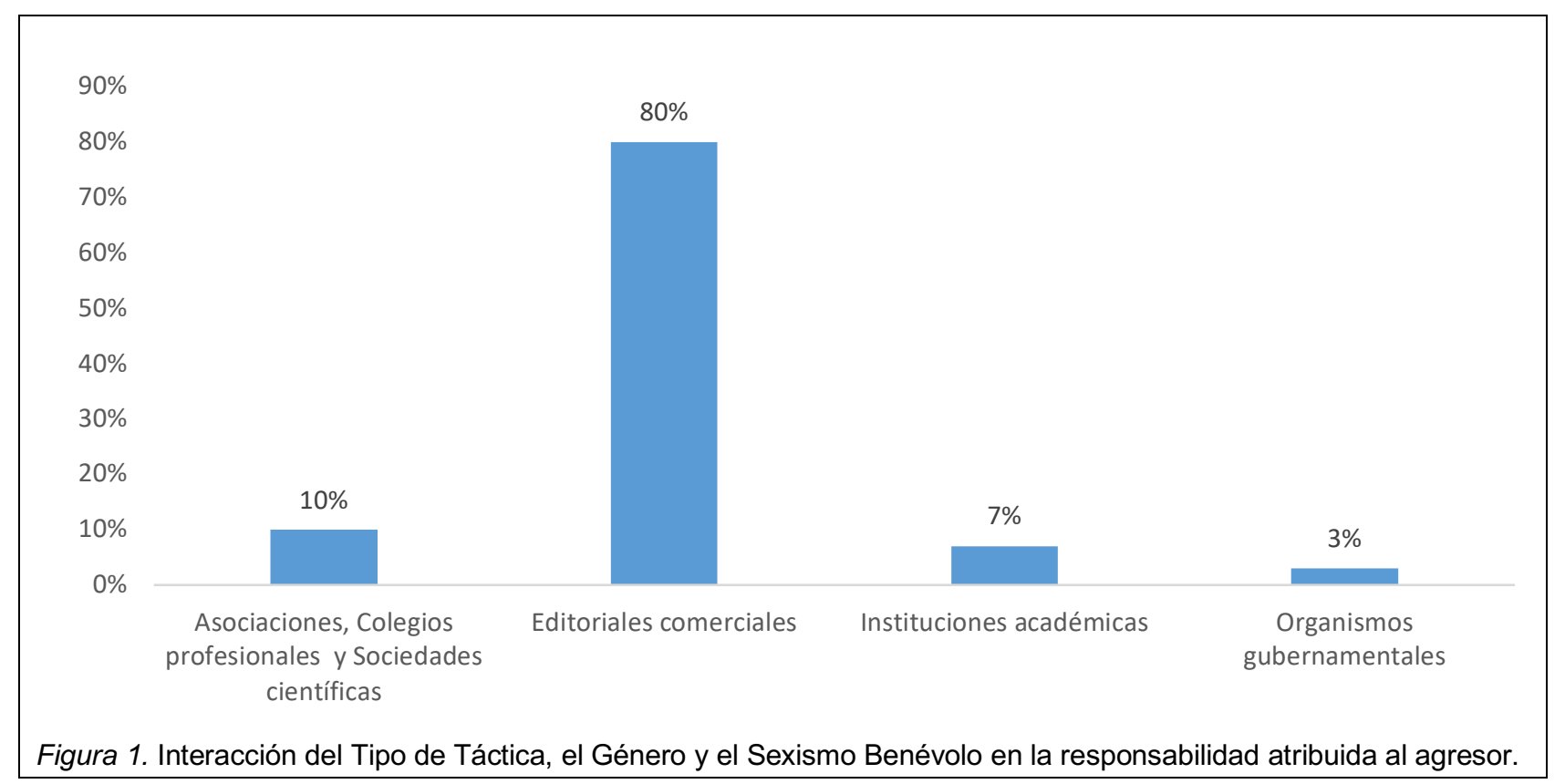

\section{Idiomas de publicación}

La mayor parte de las revistas de psicología jurídica publican sus trabajos en inglés $(90 \%, 27$ revistas). Solamente hay cuatro revistas $(10 \%)$ que publican también en otros idiomas. En este caso se trata de una revista española, que publica en español e inglés, una suiza que publica en francés y, por último, una revista croata y otra eslovena, que publican respectivamente en croata y en esloveno.

\section{Las instituciones editoras de las revis- tas de psicología jurídica}

Cuatro grupos de instituciones publican las revistas de psicología jurídica: las Editoriales comerciales, las Asociaciones, Colegios Profesionales y Sociedades Científicas, las Instituciones académicas y los Organismos gubernamentales. Tal como se muestra en la Figura 1, la mayor parte de las revistas son editadas por editoriales comerciales $(80 \%)$ y Asociaciones, Colegios Profesionales y Asociaciones Científicas (10\%).

\section{Análisis de las categorías temáticas}

Las revistas se han clasificado en 22 categorías temáticas. En JCR, se han clasificado en 10 categorías temáticas y en SJR se han clasificado en 17. Solamente hay cinco categorías temáticas que utilizan tanto las bases de datos JCR como las SJR para clasificar a las revistas analizadas, que son Law, Psychology Applied, Psychology Clinical, Psychology Social y Social Work. Tal como hemos indicado anteriormente, una misma revista, puede estar incluida en varias categorías simultáneamente. En las Tablas 2 y 3 se puede ver la relación de categorías temáticas en las que han sido clasificadas las revistas de psicología jurídica en cada una de las bases de datos. Destaca especialmente Criminology \& Penology en el JCR por aglutinar el mayor número de revistas, mientras que en la base de datos SJR destacan Law, Pathology of Forensic Medicine y Psychology Applied. 
Tabla 2

\section{Categorías temáticas en el JCR}

\begin{tabular}{lc}
\hline Categorías & No $^{\circ}$ revistas \\
\hline Criminology and Penology & 23 \\
Law & 6 \\
Psychology Multidisciplinary & 6 \\
Family Studies & 4 \\
Psychology Applied & 4 \\
Psychology Clinical & 4 \\
Psychiatry & 4 \\
Psychology Social & 2 \\
Social Work & 2 \\
Sociology & 2 \\
\hline
\end{tabular}

Tabla 3

Categorías temáticas en el SJR

\begin{tabular}{lc}
\hline Categorias & $\begin{array}{c}\mathbf{N}^{\mathbf{0}} \\
\text { revistas }\end{array}$ \\
\hline Law & $\mathbf{1 5}$ \\
Pathology of Forensic Medicine & $\mathbf{1 4}$ \\
Psychology Applied & $\mathbf{1 3}$ \\
Psychiatry and Mental Health & $\mathbf{9}$ \\
Psychology Social & $\mathbf{8}$ \\
Psychology (Miscellanous) & $\mathbf{7}$ \\
Psychology Clinical & $\mathbf{5}$ \\
Sociology and Political Science & $\mathbf{4}$ \\
Arts \& Humanities (Miscellaneous) & $\mathbf{3}$ \\
Health (Social Science) & $\mathbf{3}$ \\
Developmental and Education Psychology & $\mathbf{2}$ \\
Social Work & $\mathbf{2}$ \\
Cultural Studies & $\mathbf{1}$ \\
Education & $\mathbf{1}$ \\
Health Professions (Miscellaneous) & $\mathbf{1}$ \\
Medicine (Miscellanous) & $\mathbf{1}$ \\
Public Health, Environmental and & $\mathbf{1}$ \\
Occupational Health & \\
\hline
\end{tabular}

\section{Visibilidad e impacto}

La utilización de indicadores bibliométricos, que cuantifican y analizan la investigación a través de las publicaciones científicas es cada vez más frecuente en la actualidad. Entre los diferentes indicadores utilizado, uno que cada vez tiene mayor influencia es el del factor impacto. La idea de medir el "impacto" producido por un autor, una revista o un grupo en el mundo científico mediante el recuento de las citas que sus publicaciones han tenido en la literatura científica cada vez tiene más trascendencia. Aunque las revistas de "corriente principal" dan mayores garantías al investigador de que su trabajo no pase inadvertido, y aumentan el valor de circulación de los resultados de investigación y les aseguran una mayor cobertura en bases de datos y un mayor potencial de lectores, realmente es el análisis de citas y el factor de impacto los que se ha convertido en el auténtico patrón oro (Torres-Salinas y Cabezas-Clavijo, 2013) en la evaluación de revistas, investigadores, profesores e investigadores, becarios, universidades, proyectos, grupos de investigación y programas de doctorado (Olivas-Ávila y Musi-Lechuga, 2010; Ortiz-de-Urbina-Criado y MoraValentín, 2013; Torres-Salinas, Delgado-López-Cózar, García-Moreno-Torres y Herrera, 2011; Torres-Salinas, Delgado-López-Cózar y Jiménez-Contreras, 2009).

Cada vez es más importante para los investigadores de algunas disciplinas científicas, como la psicología, conocer qué cuartil o posición ocupa una revista dentro de su categoría temática, principalmente, en las bases de datos JCR. La presión por llegar a publicar en las revistas incluidas en los dos primeros cuartiles ha empujado a la falsificación y manipulación de las investigaciones y los currículos de los investigadores. La situación creada ha generado, y no solo en España, una auténtica "cultura de publicación científica en revistas con factor de impacto" en evidente detrimento de la publicación de libros, e incluso ha cambiado el formato tradicional de las tesis doctorales (Buela-Casal, 2014), aunque se trate de una medida altamente polémica (Buela-Casal y Zych, 2012), sin embargo determinar cuáles son las mejores revistas de una determinada área científica o especialidad es una tarea muy compleja (Osca-Lluch, 2005). En la tabla 4 se muestra la relación de revistas de psicología jurídica que actualmente están incluidas en los JCR o SJR de la edición del 2015. Algunas de las revistas de psicología jurídica, que están clasificadas en diferentes categorías temáticas en las dos bases de datos consultadas, ocupan la misma posición en ambas bases de datos y en todas las categorías en las que se encuentran incluidas. Esto sucede en el caso de las revistas Journal of Research in Crime and Delinquency, Journal of Criminal Justice, Journal of Research in Crime and Delinquency, Law and 
Tabla 4

Cuartiles de las revistas de psicología jurídica incluidas en las bases de datos JCR y SJR del año 2015

\begin{tabular}{|c|c|c|c|c|c|c|c|c|}
\hline \multirow{2}{*}{ Revistas } & \multicolumn{4}{|c|}{ JCR } & \multicolumn{4}{|c|}{ SJR } \\
\hline & 2015 & 2015 & 2105 & 2015 & 2105 & 2105 & 2015 & 2105 \\
\hline Aggression and Violent Behavior & Q1 & Q2 & & & Q1 & Q1 & Q1 & \\
\hline American Journal of Forensic Psychology & & & & & Q4 & Q4 & & \\
\hline Anuario de Psicologia Juridica & & & & & Q4 & Q4 & Q4 & \\
\hline Behavioral Sciences and the Law & Q2 & Q3 & & & Q1 & Q1 & Q2 & Q2 \\
\hline British Journal of Criminology & Q1 & & & & Q1 & Q2 & Q2 & Q2 \\
\hline Criminal Behaviour and Mental Health & Q2 & Q3 & & & Q2 & Q2 & Q2 & \\
\hline Criminal Justice and Behavior & Q2 & Q3 & & & Q1 & Q1 & Q1 & \\
\hline Deviance et Societe & Q4 & Q4 & & & Q2 & Q3 & Q3 & Q4 \\
\hline Deviant Behavior & Q3 & Q3 & Q4 & & Q2 & Q1 & Q2 & Q1 \\
\hline $\begin{array}{l}\text { European Journal of Psychology Applied to Legal } \\
\text { Context }\end{array}$ & Q2 & Q3 & & & Q1 & Q3 & & \\
\hline Homicide Studies & Q2 & & & & Q2 & Q2 & Q3 & \\
\hline International Journal of Offender Therapy and & Q2 & Q3 & & & Q2 & Q2 & Q2 & \\
\hline Comparative Criminology & & & & & & & & \\
\hline Journal of Aggression, Maltreatment and Trauma & Q4 & Q4 & Q4 & Q4 & Q2 & Q2 & Q3 & \\
\hline Journal of Criminal Justice & Q1 & & & & Q1 & Q1 & Q1 & Q1 \\
\hline Journal of Forensic Practice & & & & & Q2 & Q3 & Q3 & Q4 \\
\hline Journal of Forensic Psychiatry and Psychology & Q4 & Q4 & & & Q2 & Q3 & & \\
\hline Journal of Forensic Psychology Practice & Q4 & Q4 & & & Q3 & Q4 & & \\
\hline Journal of Interpersonal Violence & Q2 & Q2 & Q2 & & Q1 & Q1 & & \\
\hline Journal of Investigative Psychology and Offender & Q4 & Q4 & & & Q3 & Q3 & & \\
\hline & & & & & & & & \\
\hline Journal of Research in Crime and Delinquency & Q1 & & & & Q1 & & & \\
\hline Law and Human Behavior & Q1 & Q1 & & & Q1 & Q1 & Q1 & Q1 \\
\hline Legal and Criminological Psychology & Q1 & Q1 & Q2 & & Q1 & Q2 & & \\
\hline Ljetopis Socijalnog Rada & Q4 & & & & Q4 & Q3 & Q4 & Q3 \\
\hline Psychiatry Psychology and Law & Q3 & Q3 & Q3 & Q4 & Q2 & Q3 & Q3 & Q3 \\
\hline Psychology of Violence & Q1 & Q1 & Q1 & & Q1 & Q1 & Q1 & \\
\hline Psychology, Crime \& Law & Q2 & Q2 & Q3 & & Q2 & Q1 & Q2 & \\
\hline Revija za Kriminalistiko in Kriminologijo & Q4 & & & & Q4 & Q4 & Q4 & \\
\hline Sexual Abuse-A Journal of Research and Treatment & Q2 & Q1 & & & Q1 & Q1 & & \\
\hline Trauma, Violence and Abuse & Q1 & Q1 & Q1 & & Q1 & Q1 & Q1 & Q1 \\
\hline Youth Violence and Juvenile Justice & Q1 & & & & Q1 & Q1 & Q1 & \\
\hline
\end{tabular}

Human Behavior, Trauma, Psychology Violence, Violence and Abuse y Youth Violence and Juvenile Justice, que están clasificadas en todas las categorías temáticas de las bases de datos en el cuartil 1. Sin embargo, se observa que lo más frecuente ( 24 revistas), es que una revista, ocupe diferentes posiciones diferentes y, por lo tanto, diferente cuartil, dependiendo de la categoría temática en la que ha sido clasificada.

\section{Discusión}

Es apresurado hacer un balance definitivo de la investigación en psicología jurídica en la actualidad, pero siendo optimistas y viendo el estado de las revistas de psicología que publican trabajos de jurídica, podría decirse que la consolidación de la psicología jurídica está en marcha. Este trabajo revela que, aunque no existe uan categoría temática especifica de psicología jurídica en las bases de datos consultadas. Sin embargo, hay suficiente 
número de revistas, que reúnen las condiciones de estar clasificadas en categorías temáticas de psicología y jurídica, que viene a ser un indicador de la consolidación que va teniendo esta disciplina.

De acuerdo con los datos obtenidos, de las 30 revistas que cumplen los criterios en las Bases de Datos consultadas, 27 en JCR (SCI y SSCI) y 30 en SJR, sólo 3 de ellas están fuera de JCR (American Journal of Forensic Psychology, Anuario de Psicología Jurídica y Journal of Forensic Practice). De las 27 revistas incluidas en el JCR, 11 de ellas no están clasificadas en ninguna de las categorías temáticas de psicología, lo que no las tornaría, en principio, atractivas para psicólogos jurídicos y forenses, lo que dejaría en 16 el número potencial de targets para quienes investigan y /o trabajan en el ámbito.

Las 30 revistas se han clasificado en 22 categorías temáticas, una muy elevada dispersión. Las 27 revistas incluidas en JCR se han clasificado en 10 categorías temáticas, y las 30 incluidas en SJR se han clasificado en 17. Solamente hay cinco categorías temáticas que utilizan tanto las bases de datos JCR como las SJR para clasificar a las revistas analizadas, que son Law $(6+15)$, Psychology Applied (4+13), Psychology Clinical (4+5), Psychology Social (2+8) y Social Work (2+2). Criminology and Penology en JCR con 23 revistas y Pathology of Forensic Medicine en SJR con 14 son las categorías propias en las que se clasifica un mayor número de revistas.

Un $80 \%$ de las revistas las publican editoriales comerciales, un $10 \%$ Asociaciones, Colegios Profesionales y Asociaciones Científicas, un $7 \%$ instituciones académicas y un $3 \%$ organismos gubernamentales. Un aspecto que hay que destacar es que, aunque en conjunto, las revistas proceden de ocho países diferentes, solamente dos países son los que editan el $77 \%$ de las publicaciones: Estados Unidos y Reino Unido.

Respecto al idioma de publicación de los trabajos, hay que mencionar que las revistas de lengua materna inglesa son 24 (12 UK, 11 EU y uno Australia), y seis proceden de países europeos con lenguas nativas diferentes (Croacia, Eslovenia, España, Holanda y Suiza), pero dos de ellas, una española y la holandesa también publi- can en inglés. De esta forma, se observa que solo cuatro revistas publican en lengua propia, e incluso una lo hace en español e inglés, una situación que otorga un peso demoledor al inglés, y recuerda la época medieval en la que el latín era la lengua de la cultura y las demás lenguas no existían en el mundo de la ciencia.

Las revistas de mayor atractivo para los investigadores serían sin duda: Journal of Criminal Justice; Journal of Research in Crime and Delinquency; Law and Human Behavior; Psychology of Violence; Trauma, Violence and Abuse; y Youth Violence and Juvenile Justice, clasificadas en todas las categorías temáticas de las bases de datos (JCR y SJR) en el cuartil 1. También sería muy recomendable el British Journal of Criminology (Q1 en JCR y Q1 y Q2 en SJR). No obstante, lo más frecuente (24 revistas de 30 ), es que una revista, ocupe posiciones diferentes y, por lo tanto, distinto cuartil, dependiendo de la categoría temática en la que hubiera sido clasificada.

Sin embargo, del total de las publicaciones analizadas, desde el punto de vista de los criterios de evaluación de la Comisión Nacional Evaluadora de la Actividad Investigadora (CNEAI), encargada de evaluar periodos sexenales de actividad investigadora de los profesores universitarios y del personal de las escalas científicas del $\mathrm{CSIC}^{1}$, solamente 18 revistas de psicología jurídica, de las analizadas en este trabajo, las incluidas en el JCR, servirían para la acreditación de investigadores españoles. Las revistas que cumplen estos requisitos son: British Journal of Criminology (Q1), Journal of Criminal Justice (Q1), Journal of Research in Crime and Delinquency (Q1), Law and Human Behavior (Q1, Q1), Psychology of Violence (Q1, Q1, Q1), Trauma, Violence and Abuse (Q1, Q1, Q1), Youth Violence and Juvenile Justice (Q1), Aggression and Violent Behavior (Q1, Q2), Legal and Criminological Psychology (Q1, Q1, Q2), Sexual Abuse-A Journal of Research and Treatment (Q2, Q1), Behavioral Sciences and the Law (Q2, Q3), Criminal Behaviour and Mental Health (Q2, Q3), Criminal

\footnotetext{
${ }^{1}$ Resolución de 30 de noviembre de 2016, de la Secretaría de Estado de Educación, Formación profesional y Universidades, por la que se fija el procedimiento y plazo de presentación de solicitudes de evaluación de la actividad investigadora a la Comisión Nacional Evaluadora de la Actividad Investigadora. Publicado en: «BOE» núm. 291, de 2 de diciembre de 2016, páginas 84669 a 84674 .
} 
Justice and Behavior (Q2, Q3), European Journal of Psychology Applied to Legal Context (Q2, Q3), Homicide Studies (Q2), International Journal of Offender Therapy and Comparative Criminology (Q2, Q3), Journal of Interpersonal Violence (Q2, Q2, Q2), Psychology, Crime \& Law (Q2, Q2, Q3).

El análisis de las revistas de psicología jurídica demuestra la existencia de una disciplina que, en España, cada día está más consolidada pero que necesita contar con unas revistas especializadas más consolidadas, con el propósito de que estos pueden publicar los resultados de sus investigaciones en revistas nacionales de su especialidad. Actualmente, las pocas opciones que tienen los especialistas de la materia para publicar trabajos en revistas de su disciplina que les sirvan para que se reconozcan sus méritos académicos, contribuye a que estos deriven sus trabajos a revistas de carácter general o multidisciplinar, lo que contribuye a restar visibilidad a los trabajos o que estos pasen desapercibidos para el resto de especialistas en la materia, que consultan para su formación o actualización la información bibliográfica en revistas especializadas.

\section{Referencias}

Álvarez-García, D., Barreiro-Collazo, A., Núñez, J. C. y Dobarro, A. (2016). Validity and Reliability of the Cyber-Aggression Questionnaire for Adolescents (CYBA). European Journal of Psychology Applied to Legal Context, 8, 69-77. https://doi.org/10.1016/j.ejpal.2016.02.003

Amado, B. G., Arce, R. y Fariña, F. (2015). Undeutsch Hypothesis and Criteria Based Content Analysis: A Meta-Analytic Review. European Journal of Psychology Applied to Legal Context, 7, 3-12. https://doi.org/10.1016/j.ejpal.2014.11.002

Amado, B. G., Arce, R., Farina, F. y Vilariño, M. (2016). Criteria-Based Content Analysis (CBCA) Reality Criteria in Adults: A Meta-Analytic Review. International Journal of Clinical and Health Psychology, $16 \quad$ 201-210. https://doi.org/10.1016/j.ijchp.2016.01.002

Arce, R. (2005). La construcción de la psicología jurídica en Europa y su estatus actual [La construcción de la psicología jurídica en Europa y su estatus actual]. En R. Abrunhosa y C. Machado (Eds.), Manual de psicología jurídica (pp. 103-114). Coimbra, Portugal: Quarteto.

Arce, R., Fariña, F. y Fraga, A. (2000). Género y formación de juicios en un caso de violación [Gender and Juror Judgment Making in a Case of Rape]. Psicothema, 12(4), 623-628. Recuperado de http://www.psicothema.com/pdf/381.pdf

Arce, R., Fariña, F. y Vilariño, M. (2015). Daño psicológico en casos de victimas de violencia de género: Estudio comparativo de la evaluación forense [Psychological Injury in Intimate Partner Violence Cases: A Contrastive Analysis of Forensic Measures]. Revista Iberoamericana de Psicología y Salud, 6 , 72-80. 
Buela-Casal, G. (2014). Pathological Publishing: A new Psychological Disorder with Legal Consequences? European Journal of Psychology Applied to Legal Context, 6, 91-97. https://doi.org/10.1016/j.ejpal.2014.06.005

Buela-Casal, G., Bermúdez, M. P., Sierra, J. C., GuillénRiquelme, A. y Quevedo-Blasco, R. (2015). Productividad y eficiencia en investigación por comunidades autónomas españolas según la financiación (2012) [Productivity and Efficiency in Research for Spanish Regions by Funding (2012)]. Revista Iberoamericana de Psicología y Salud, 6, 110. https://doi.org/10.1016/S2171-2069(15)70001-3

Buela-Casal, G. y Zych, I. (2012). What Do the Scientists Think about the Impact Factor? Scientometrics, $\quad 92, \quad 281-292$. https://doi.org/10.1007/s11192-012-0676-y

Cala, J., Trigo, M. E. y Saavedra, F. J. (2016). Women's Disengagement from Legal Proceedings for Intimate Partner Violence: Sociodemographic and Psychological Variables. European Journal of Psychology Applied to Legal Context, 8, 35-42. https://doi.org/10.1016/j.ejpal.2015.10.002

Contreras, L. y Cano, M. D. (2016). Child-to-parent Violence: The Role of Exposure to Violence and its Relationship to Social-Cognitive Processing. European Journal of Psychology Applied to Legal Context, $\quad 8, \quad 43-50$. https://doi.org/10.1016/j.ejpal.2016.03.003

González-Sala, F., Osca-Lluch, J., Tortosa Gil, F. y Peñaranda Ortega, M. (2017). Characterization of Legal Psychology through Psychology Journals included in Criminology \& Penology and Law Categories of Web of Science. Anales de Psicología, 33, 411-416. https://doi.org/10.6018/analesps.33.2.262591

Navarrete-Cortés, J., Quevedo-Blasco, R., ChaichioMoreno, J. A., Ríos, C. y Buela-Casal, G. (2009). Análisis cuantitativo por países de la productividad en psicología de revistas en la Web of Science
[Quantitative Analysis by Country of Psychological Material Published in Journals in the Web of Science]. Revista Mexicana de Psicología, 26, 131$143 . \quad$ Recuperado de http://www.redalyc.org/html/2430/243016315001/

Olivas-Ávila, J. y Musi-Lechuga, B. (2010). Análisis de la producción de los profesores funcionarios de Psicología en España en artículos de revistas de la Web of Science [Analysis of the Production of Psychology Professors in Spain in Journal Articles of the Web of Science]. Psicothema, 22, 909-916. Recuperado de https://www.unioviedo.es/reunido/index.php/PST/ar ticle/viewFile/8970/8834

Ortiz-de-Urbina-Criado, M. y Mora-Valentín, E. (2013). El sistema de acreditación del profesorado a través del Programa ACADEMIA: Evolución y cambios. [The National Accreditation System of University Professors through The ACADEMIA Program: Evolution and Changes]. Revista Española de Documentación Científica, 36(1), 1-8. https://doi.org/10.3989/redc.2013.1.971

Osca-Lluch, J. (2005). Some Considerations on the Use of the Impact Factor of Scientific Journals as a Tool to Evaluate Research in Psychology. Scientometrics, $\quad 65$, 189-197. https://doi.org/10.1007/s11192-005-0267-2

Osca-Lluch, J. (2012). Aspectos regionales de las revistas españolas de ciencias sociales y humanidades: calidad y visibilidad internacional [Regional Aspects of Social Sciences Spanish Journals: Quality and International Visibility]. Biblio 3 W. Revista Bibiográfica de Geografia y Ciencias Sociales, XVII, 998. Recuperado de http://www.ub.edu/geocrit/b3w-998.htm

Quiñones, E., Peñaranda, M., López, J. J. y Tortosa, F. (2004). Social and Personality Psychology as reflected in Authorships and Citations of Journal of Personality and Social Psychology, 1965-2000. Journal of Personality and Social Psychology, 86, 435-452. 